\title{
ON CONTRACTIVE MAPPINGS IN UNIFORM SPACES
}

\author{
W. J. KAMMERER AND R. H. KASRIEL
}

Introduction. Let $(X, \rho)$ be a metric space and let $f$ be a mapping of $X$ into $X$. The mapping $f$ is said to be $\epsilon$-contractive, if there exists an $\epsilon>0$ such that $\rho(f x, f y)<\rho(x, y)$ whenever $0<\rho(x, y)<\epsilon$. Among the results established by M. Edelstein in [2] were the following:

1.1. If $X$ is a compact metric space and $f$ an $\epsilon$-contractive mapping on $X$, then there exists a periodic point $\xi$ (i.e., for some positive integer $\left.k, f^{k}(\xi)=\xi\right)$.

1.2. If in addition, $X$ is $\epsilon$-chainable, then $\xi$ is a unique fixed point and $\xi=\lim _{n \rightarrow \infty} f^{n}(x)$ for each $x \in X$.

It is the purpose of this note to point out that these results can be extended to the more general setting of uniform spaces. Toward this end there are listed, in 1.3 through 1.6, some definitions and results from [1], which will be referred to later.

Let $(X, \mathfrak{u})$ be a uniform space and let $\&$ be a basis for the uniformity. (See Kelley [4, Chapter 6].)

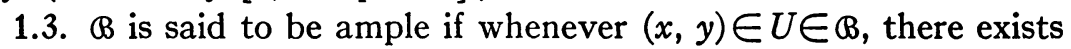

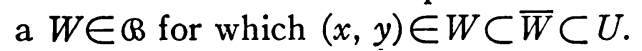

1.4. A function $f: X \rightarrow X$ is said to be weakly $B$-contractive, if $(f x, f y) \in U$ whenever $(x, y) \in U \in \beta$.

1.5. A function $f: X \rightarrow X$ is said to be weakly $B$-expansive if $(x, y) \in U$ whenever $(f x, f y) \in U \in ß$.

1.6. Let $B$ be an open, ample basis for the totally bounded Hausdorff uniform space $(X, \mathfrak{u})$ and let $f$ map $X$ onto $X$. If $f$ is weakly B-contractive then $f$ is also weakly $B$-expansive. (See [1, Theorem 2.1].)

As a historical note, it should be pointed out that the metric version of 1.6 was obtained by Edrei [3] and an extension similar to 1.6 was obtained by Rhodes [5] for uniform spaces.

In addition we introduce the following definitions.

1.7. Let $(X, \mathfrak{u})$ be a uniform space and let $\leftrightarrow$ be a basis for $\mathcal{u}$. A function $f: X \rightarrow X$ is said to be $B$-contractive, provided that for

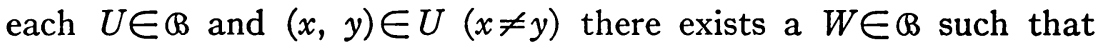
$(f x, f y) \in W \subset U$ and $(x, y) \notin W$.

1.8. Let $Q$ be a basis for the uniformity for a space $X$ and let $U \in B$. Then a $U$-chain is any finite set of points $x_{0}, x_{1}, \cdots, x_{n}$ in $X$ such that $\left(x_{i-1}, x_{i}\right) \in U, i=1,2, \cdots, n$. We shall say in such a case that $x_{0}$ and $x_{n}$ are joined by a $U$-chain.

Received by the editors August 3, 1962 and, in revised form, January 3, 1963. 
The principal result of this paper is stated next.

1.9. Theorem. Let $(X, \mathcal{u})$ be a Hausdorff uniform space and let $B$ be an open ample basis for the uniformity of $X$. If $f: X \rightarrow X$ is $B$ contractive and is such that the image of $X$ under some iterate of $f$ is compact, then

(a) The set of periodic points in $X$ is a nonempty finite set $A=\left\{x_{0}, x_{1}, \cdots, x_{n}\right\}$ so that for some positive integer $p, f^{p}\left(x_{i}\right)=x_{i}$ for each $x_{i}$ in $A$. Furthermore, for each $x$ in $X$, there exists an $x_{i} \in A$ such that $\lim _{n \rightarrow \infty} f^{p n}(x)=x_{i}$.

(b) Suppose $X$ is $U$-chainable, $U \in ß$. Then $A$ reduces to a single point. [Hence $f$ possesses a unique fixed point $x_{0}$ and for each $x \in X$, $\lim _{n \rightarrow \infty} f^{n}(x)=x_{0}$.]

2. Proof of Theorem 1.9. Theorem 1.9 will be established by proving a sequence of lemmas, in each of which $X$ is taken to be a compact Hausdorff uniform space and $f$ a continuous mapping of $X$ into $X$. Define $X=X_{0}, X_{n}=f\left(X_{n-1}\right)$ for $n=1,2, \cdots$ and $A=\cap X_{n}$. Since $\left\{X_{n}: n=0,1, \cdots\right\}$ is a nested sequence of nonempty compact sets, it follows that $A$ is also a nonempty compact set.

2.1. $f(A)=A$.

The straightforward proof of 2.1 is omitted.

2.2. If $B$ is an open ample basis and $f: X \rightarrow X$ is $B$-contractive, then $A$ consists of a finite number of points.

Proof. The collection of sets $B^{*}=\{U \cap(A \times A): U \in B\}$ forms an open ample basis for the relative uniformity of $A$. Hence the restriction of $f$ to $A, f \mid A$, is $B^{*}$-contractive and hence weakly $B^{*}$-contractive. Since $A$ is compact, $A$ is complete and totally bounded. By 1.6, $f \mid A$ is also weakly $B^{*}$-expansive. Let $U \in B^{*}$. Then for distinct $x$ and $y$ in $A$, one has $(x, y) \notin U$. For if this were not the case, there would exist a $W \in B^{*}$ such that $W \subset U,(x, y) \in U-W$ and $(f x, f y)$ $\in W$. But since $f$ is also weakly $B^{*}$-expansive, $(x, y)$ must be in $W$. This, however, is a contradiction.

Let $U$ be any element in $B^{*}$. Then $U[x]=\{y:(x, y) \in U\}$ is an open set in the relative topology of $A$. By the previous paragraph, the set $U[x]$ contains only $x$. Since $A$ is compact there exists a finite number of sets of the form $U[x]$ with $x \in A$, which cover $A$. Thus $A$ is a finite set.

2.3. With the same hypothesis as in 2.2 , there exists a positive integer $p$ such that $f^{p}(a)=a$ for each $a \in A$. Furthermore, every periodic point of $f$ is in $A$.

The results follow easily from the fact that $A$ is a finite set and $f(A)=A$. 
2.4. Under the same hypothesis as in 2.2, the sequence $\left\{f^{p n}(X): n=0,1,2, \cdots\right\}$ converges to some element of $A$.

Proof. Let $\bar{x}$ be any cluster point of the sequence $\left\{f^{p n}(x)\right.$ : $n=1,2,3, \cdots\}$. The point $\bar{x}$ must be an element of $A$. For if not, there exists an $n_{0}$ for which $\bar{x} \in X_{i}$ for all $i \geqq n_{0}$. Since $X_{n_{0}}$ is closed, there exists a $V \in ß$ such that $V[\bar{x}] \cap X_{n_{0}}=\varnothing$. But $V[\bar{x}]$ contains infinitely many points of the form $\left\{f^{p n}(x)\right\}$ giving a desired contradiction. To show that $\lim f^{p n}(x)=\bar{x}$, let $U \in \mathbb{B}$. There exists an $n$ such that $\left(\bar{x}, f^{p n}(x)\right) \in U$. Since $f^{p}$ is also $B$-contractive and $f^{p}(\bar{x})=\bar{x}$ one has $\left(\bar{x}, f^{p(n+1)}(x)\right) \in U$. Hence $\lim f^{p n}(x)=\bar{x}$.

2.5. Suppose, in addition to the hypothesis of $2.4, X$ is $U$-chainable. Then $A$ consists of a single point. Hence $f$ possesses a unique fixed point.

Proof. Suppose $(x, y) \in U \in B$. Then by 1.3 , there exists a $V \in \mathbb{B}$ such that $(x, y) \in V \subset \bar{V} \subset U$. From 1.4, it follows that $\left(f^{p n}(x), f^{p n}(y)\right)$ $\in V$ for $n=1,2, \cdots$ and from the proof of 2.4 it follows that $\left(\lim _{n \rightarrow \infty} f^{p n}(x), \lim _{n \rightarrow \infty} f^{p n}(y)\right) \in \bar{V} \cap(A \times A) \subset U \cap(A \times A)$. By making use of this, it is easy to show that if $X$ is $U$-chainable, so is $A$. But by the argument as in the proof of $2.2, A$ can only be $U$-chainable if $A$ consists of a single point.

2.6. If $A$ consists of a single point $a$, then $\lim _{n \rightarrow \infty} f^{n}(x)=a$.

Proof. 2.6 follows from the proof of 2.4 upon setting $p=1$.

\section{BIBLIOGRAPHY}

1. T. A. Brown and W. W. Comfort, New methods for expansion and contraction maps in uniform spaces, Proc. Amer. Math. Soc. 11 (1960), 483-486.

2. Michael Edelstein, On fixed and periodic points under contractive mappings, J. London Math. Soc. 37 (1962), 74-79.

3. Albert Edrei, On mappings which do not increase small distances, Proc. London Math. Soc. 2 (1952), 272-278.

4. John L. Kelley, General topology, Van Nostrand, New York, 1955.

5. F. Rhodes, $A$ generalization of isometries to uniform spaces, Proc. Cambridge Philos. Soc. 52 (1956), 399-405.

Georgia Institute of Technology 